\title{
HEART-TYPE FATTY ACID BINDING PROTEIN VERSUS CARDIAC TROPONIN I IN EARLY DETECTION OF MYOCARDIAL INJURY INDUCED BY SCORPION STING.
}

\author{
Amr R. Zaki*, Mostafa Abutaleb Ahmed** \\ Department of forensic medicine and clinical toxicology, Faculty of Medicine, Beni Suef \\ University*, Department of forensic medicine and clinical toxicology, Faculty of Medicine, \\ Minia University**, Egypt
}

\begin{abstract}
Background: Scorpion envenomation is an important public health hazard in tropical and sub- tropical regions. Envenomation by scorpions can result in a wide range of clinical effects, including, cardiotoxicity, neurotoxicity and respiratory dysfunction. Biomarkers play an important role in the early diagnosis and management of patients with the cardiotoxicity related to scorpion sting. Diagnosis of myocardial injury at an early stage in the emergency department is often difficult. A recently proposed biomarker, heart fatty acid binding protein (H-FABP) has been found to appear in the circulation superior to that of cardiac troponins in the early hours of scorpion sting. Objective: The objective of this study was to evaluate the clinical reliability of heart-type fatty acid-binding protein (h-FABP) in identifying patients with the cardiotoxicity in the early hours following scorpion sting. Methods: troponin I (in laboratory) and H-FABP were performed on 246 patients who presented with scorpion sting with duration since onset ranging from 20 minutes to 2 hours. Results: Data of the 246 patients were analyzed. H-FABP had a higher sensitivity of $76.40 \%$ and a specificity of $96.10 \%$ compared with $58.30 \%$ and $98 \%$ for troponin I respectively (at first 6 hours).Conclusion: H-FABP was found to be a better biomarker of cardiac necrosis in the early hours in the diagnosis of non-conclusive ECG in patients with scorpion sting.
\end{abstract}

Keywords: Scorpion ; H-FABP; troponin I; myocarditis; ROC

\section{INTRODUCTION}

Scorpion sting is a distressing endemic public health problem in several developing countries. Complications of Scorpion envenomation are cardiotoxicity, neurotoxicity and respiratory distress (Himmatrao and Pramodini, 2012).

These conditions require early diagnosis and ICU care from few hours to days. High morbidity and mortality occur due to delay in recognition, several studies in recent years revealed the accuracy and efficiency of evaluation of these patients; using ideal blood markers for early diagnosis of myocardial injury (Bawaskar and Bawaskar, 1992).

The ideal cardiac marker should be highly sensitive and specific; rapidly rise and fall after ischemia, can be performed simply, and not affected by other organs, particularly the kidney (Abdel-Reheim, et al., 2002).

The presence of positive cardiac biomarkers indicates higher risk and worse prognosis (Ishii et al., 1997).

Early diagnosis of myocardial injury is sometimes difficult due to ambiguous electrocardiogram (ECG) changes and delayed detection of cardiac markers such as troponin and 
creatine kinase $(\mathrm{CK})$. Cardiac troponin I (cTnI) begins rising in the blood 4-6 hours post infarction. It peaks in 12-24 hours but may take weeks to return to normal (Bawaskar and Bawaskar, 1992).

Heart type fatty acid binding protein $(\mathrm{H}-\mathrm{FABP})$ is a new marker with a low molecular weight cytoplasmic protein. It is abundant in myocardial tissue; its concentration in skeletal muscle is about $10-20 \%$ of its concentration in heart muscle (Donoghue et al., 2006).

Several studies revealed that $\mathrm{H}$ FABP is a rapid indicator for evaluation of myocardial damage in patients with scorpion sting related myocarditis. It is more sensitive and becomes positive earlier than troponins (Glatz et al., 2002).

Following myocardial cell damage, this small protein diffuses much more rapidly than troponins through the interstitial space (Ayca et al., 2012).

It appears in the circulation as early as $90 \mathrm{~min}$ after the onset of the symptoms, reaching its peak within six hours and remains positive for nearly 24 hours. These features make h-FABP an excellent indicator for myocardial injury (Ghani et al., 2000).

This prospective study was undertaken to evaluate h-FABP versus troponin $I$ as a rapid indicator for assessment of myocardial damage in patients with scorpion sting and nonconclusive ECG.

\section{MATERIAL \& METHODS}

The study was conducted from January 2010 to December 2014 in Clinical toxicology Department, Faculty of Medicine, Minia university hospital. This paper highlights the diagnostic performance of h-FABP which was compared with that of troponin I, the standard test in clinical practice. Total 246 patients suffering from Scorpion sting satisfying inclusion criteria were selected. All patients had a 12-lead ECG and biochemical markers which included troponin I and h-FABP. After the initial assessment, venous blood was drawn from patients at time of presentation and at six hours from onset of sting then after $24 \mathrm{hrs}$ from the onset, into plain tubes (without anticoagulants) and the serum sample was stored at $-20{ }^{\circ} \mathrm{C}$ until biochemical parameters were determined.

\section{Inclusion criteria}

- Patients with history of scorpion sting.

- The age below 18 years for both genders.

- Time window of admission: onset of sting within 2 hours.

- Patient with signs suggestive of envenomation including local pain of varying degrees, repeated vomiting, centripetal hyperthermia, priapism, agitation and shock.

\section{Exclusion criteria}

- Above 18 years of age.

- Known renal disease (serum creatinine level $2.0 \mathrm{mg} / \mathrm{dl}$ or more): as Heart type fatty acid binding protein (H-FABP) level is usually elevated in patients with renal insufficiency, due to clearance of h-FABP through the kidney.

- Traumatic injury

- Patients who are not in position to give consent.

\section{Biochemical Analyses}

Serum level of Heart type fatty acid binding protein (h-FABP) was measured using a recently developed ELISA, Oxis Research Inc, USA. The sensitivity of h-FABP assay was 17.7 ng/ml. Serum cTnI level was determined using Immulite troponin I 
Kit which is a solid-phase, two-site chemiluminescent enzyme immunometric assay for use with the immulite automated analyzer (Cat No. LKTI, Diagn. Products Corp, USA). The detection limit of cTnI assay was approximately $\geq 0.1 \mathrm{ng} / \mathrm{ml}$. The diagnostic performance of h-FABP was compared with that of troponin I, the standard test in clinical practice. The diagnosis of myocardial injury (myocarditis) was confirmed with Echocardiography (wall motion abnormalities, $\downarrow$ ejection fraction) and positive troponin at time point $24 \mathrm{hr}$ from onset of sting. Data were analyzed for clinical sensitivity, specificity and negative predictive value.

\section{Statistical method}

The collected data were coded, tabulated, and statistically analyzed using SPSS program (Statistical Package for Social Sciences) software version 20. ROC curve analysis was used to determine area under the curve, sensitivity, specificity, positive predictive value, negative predictive value and accuracy. The level of significance was taken at $(\mathrm{P}$ value $\leq$ 0.05).

\section{RESULTS}

A total of 246 patients (144 males and 102 females) were included in this study. ECG findings (ST segment elevation in leads II, III, Avf and V5, V6) were enrolled also in the study.

Analysis of the data showed repeated troponin testing 6 hours after the onset of envenomation showed positive results in 84 patients, while Heart type fatty acid binding protein (h-FABP) diagnosed 110 stinged patients with myocardial injury as compared to 84 patients by troponin $\mathrm{I}$ in the initial 6- hour period; i.e. 26 more patients were diagnosed by h-FABP.

As shown in Table 1 , the sensitivity and specificity of troponin I for the diagnosis of myocardial injury at hospital admission were $28.5 \%$ and $100 \%$, respectively, the sensitivity and specificity of H-FABP were $45.1 \%$ and $100 \%$, which were significantly higher than the sensitivity of troponin I ( $\mathrm{P}$ $<0.001)$, but there was no statistical difference in specificity between them. Also another analysis showed that the sensitivity and specificity of troponin I for the diagnosis of myocardial injury in the initial 6 hours after the onset of sting ,were $58.59 \%$ and $98 \%$, respectively, the sensitivity and specificity of H-FABP were $76.40 \%$ and $96.10 \%$ which were significantly higher than the sensitivity of troponin I ( $\mathrm{P}<0.001)$. Sensitivity could have been higher if h-FABP was repeated as the other biochemical markers if the first test was negative. Heart type fatty acid binding protein (h- FABP) was found to have a higher negative predictive value when compared with that of troponin I. The negative predictive values of h-FABP and troponin I were $74.2 \%, \%$ and $62.5 \%$ respectively.

Receiver Operator Characteristic Curve (ROC) analysis was done to demonstrate H-FABP and troponin I levels (Fig 1, 2). From the ROC, the optimum cut-off value which H-FABP can be considered positive was found to be $17.7 \mathrm{ng} / \mathrm{ml}$, while the detection limit of cTnI assay was approximately $\geq 0.1 \mathrm{ng} / \mathrm{ml}$. The area under the ROC curves for H-FABP was observed to be $0.862(\mathrm{P}<0.001)$ and it was the highest among the markers, which was significantly greater than troponin I (0.782) 
Table (1): Comparison of Serum Levels of H-FABP with Troponin I in Different Study Groups

\begin{tabular}{|l|l|l|l|l|l|}
\hline & Sensitivity & Specificity & NPV & AUC & P value \\
\hline h-FABP 0 & $45.1 \%$ & $100 \%$ & 56.4 & 0.726 & $<0.001^{*}$ \\
\hline h-FABP 6 & $76.4 \%$ & $96.1 \%$ & 74.2 & 0.862 & $<0.001^{*}$ \\
\hline h-FABP 24 & $91.7 \%$ & $96.1 \%$ & 89.1 & 0.939 & $<0.001^{*}$ \\
\hline Troponin 0 & $28.5 \%$ & $100 \%$ & 49.8 & 0.642 & $<0.001^{*}$ \\
\hline Troponin 6 & $58.3 \%$ & $98 \%$ & 62.5 & 0.782 & $<0.001^{*}$ \\
\hline Troponin 24 & $100 \%$ & $98 \%$ & 100 & 0.990 & $<0.001^{*}$ \\
\hline
\end{tabular}

Area under the Curve (AUC),

Negative Predictive Value (NPV)

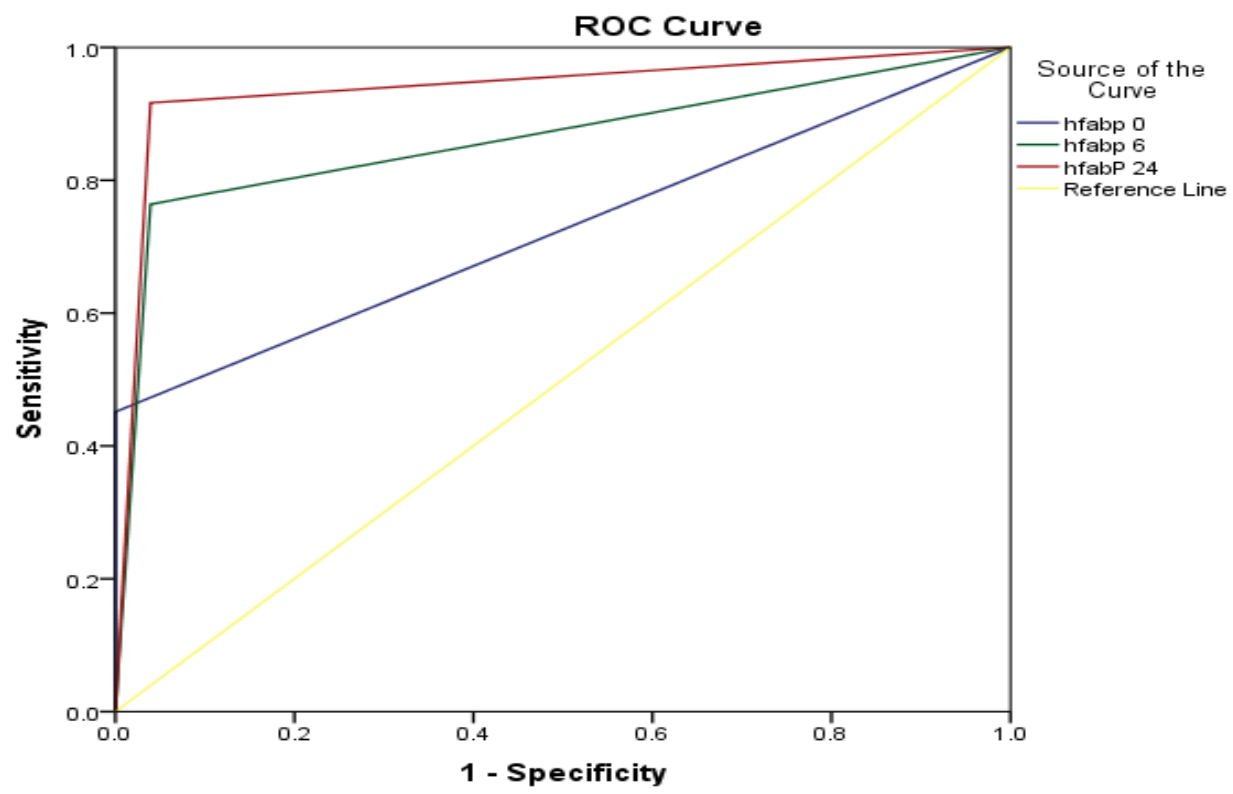

Figure (1) :Receiver Operator Characteristic Curve (ROC) Analysis for the assay of HFABP

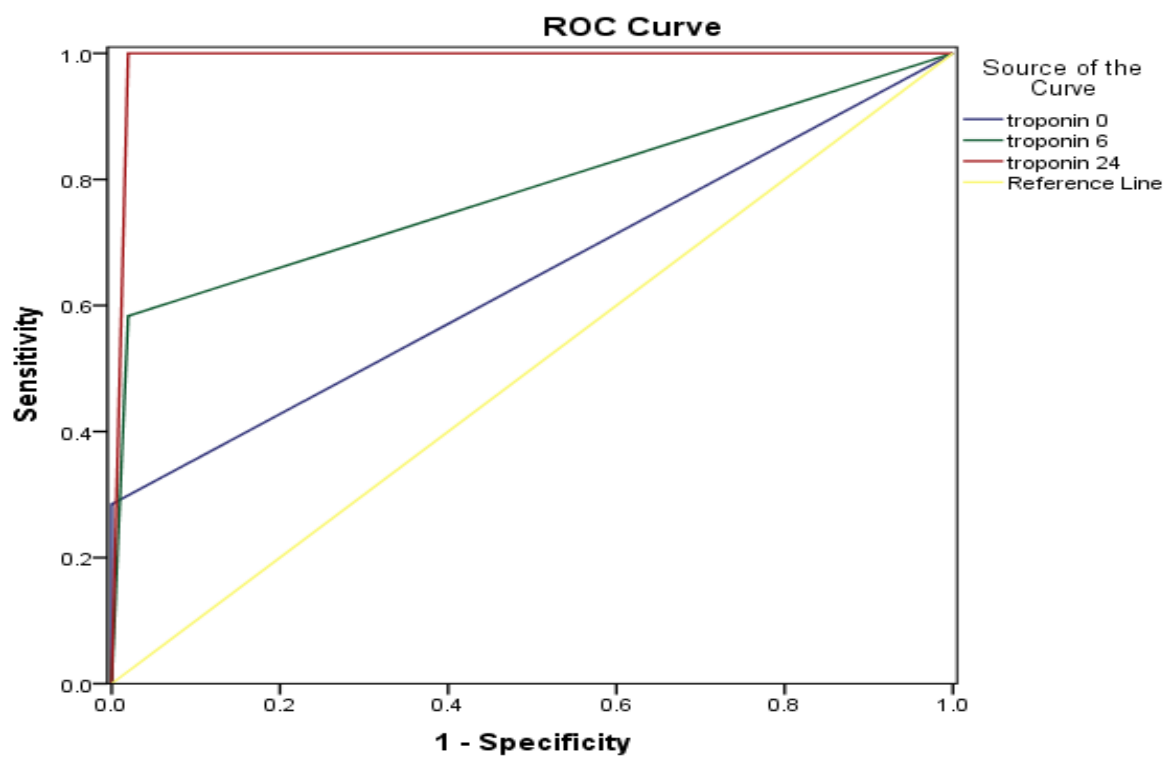

Figure (2): Receiver Operator Characteristic Curve (ROC) Analysis for the assay of cTnI. 


\section{DISCUSSION}

Scorpion venom is a complex mixture of neurotoxins, cardiotoxins, nephrotoxins and haemolysins. Venom can cause myocardial damage by several pathogenetic mechanisms, coronary artery vasospasm by release of histamine, serotonin and leukotrienes) (Ho-pang et al., 2009), toxic myocarditis by reduction of $\mathrm{Na}-$ K-ATPase (Rahav and Weiss, 1990), release of allergenic proteins causes anaphylactic shock leading to hypotension, also Scorpion venom inhibits angiotensin converting enzyme (ACE), resulting in pulmonary oedema (Bawaskar and Bawaskar, 1999).

Despite the development of assays for highly sensitive and specific biochemical markers of myocardial necrosis, myocardial injury remains a challenge. A sensitive and specific earlier marker of myocardial cell injury might be helpful in treatment decisions (Ayca et al., 2012).

The principal characteristics that would make an ideal marker for early detection of myocardial injury include, a small size of molecular marker is more rapidly released into the circulation allowing early detection of myocardial damage, also its absence in the circulation under physiological conditions, thus its detection will be abnormal with even minimal increase of the marker in the plasma and absolute specificity for the myocardium (Donoghue et al., 2006).

H-FABP's advantage is dominant in the early phase of myocardial infarction due to: its smaller size and weight, its abundance in the heart tissue and its release and clearance kinetic (Okamoto et al., 2000; Tsuji et al., 1993).
The combination of a sensitive marker such as h-FABP for early detection, with a cardio-specific marker such as troponin for later confirmation can be recommended to provide optimal diagnostic performance, to minimize the risk of false exclusions of patient with myocarditis (Katrukha et al., 1999).

Our results demonstrate that the sensitivity of $\mathrm{h}$ FABP is higher when compared to other biochemical markers troponin by approximately $18 \%$ and around the same specificity in comparison with other biomarkers in the early hours. In our study, the sensitivity of Troponin I at hospital admission was $28.5 \%$, which was very low compared with previous reports in which sensitivities ranged from $38.1 \%$ to $100 \%$, (Sayre et al., 1998; Ruzgar et al., 2006; Zarich et al., 2001; Hetland et al., 1996).

Sensitivities of H-FABP assays in patients at hospital admission and 6 hours after the onset of sting were significantly higher than those of Troponin I assays, but sensitivities of H-FABP were significantly lower than sensitivities of Troponin I 24 hours after the onset of sting, which means H-FABP are much more useful than Troponin I in patients admitted at hospital and 6hours from the onset of sting in detection of myocardial Injury. The sensitivity of H-FABP was the highest markers in patients at hospital admission and 6 hours after the onset of sting, due to its low specificity. This result is similar to the results that showed the sensitivity and specificity of H-FABP were $89 \%$ and $52 \%$ in patients admitted within 2 hours from the onset of symptoms (Seino et al., 2003), also the sensitivity and specificity of H-FABP were $89.7 \%$ and 
$72.2 \%$ within 3 hours after onset of symptoms (Okamoto et al., 2000) and the sensitivity and specificity of $\mathrm{H}$ FABP were $73 \%$ and $71 \%$ within 4 hours after onset of symptoms (McCann et al.,2008).

As our results were demonstrating an H-FABP area under the curve of 0.862 with $76.4 \%$ sensitivity and $96.1 \%$ specificity, so our results were in complete agreement with the above studies demonstrating an area under the curve of 0.965 with $87 \%$ sensitivity and $93 \%$ specificity. These results indicate the fact that H-FABP is well suited for early detection of myocarditis associating scorpion sting. Another study not agree with the above results which reported an increased area under the curve for Troponin I than H-FABP, as H-FABP levels were measured at a later period, whereas H-FABP is useful especially in the early hours after infarction (Glatz et al., 2002).

Heart-type fatty acid-binding protein (h-FABP) supplies diagnostic safety in the first and decisive hours. Heart-type fatty acid-binding protein (h-FABP) was found to be a superior biomarker of cardiac necrosis in the diagnosis of myocardial infarction due to its higher myocardial content and low plasma concentration $(1.8 \mathrm{~g} / \mathrm{l})$.

\section{CONCLUSION}

Heart-type fatty acid-binding protein (h-FABP) is a superior biomarker of cardiac necrosis in the diagnosis of scorpion sting with toxic myocarditis in patients in the early hours due to higher sensitivity and higher negative predictive value in comparison with other biomarkers, namely troponin I.

\section{ACKNOWLEDGEMENTS}

The authors wish to thank professor dr. Mohammed Abdulazeem Khalaf the president of forensic medicine and clinical toxicology department for his great and continuous assistance for us to complete our work.

\section{REFERENCES}

Abdel-Reheim AM, Zeinab MM, Hassan MM. Myocardial injury in scorpion envenomed children: significance of assessment of serum Troponin I and Interleukin8. Neuroendocrinology letters 2002;23:133-140.

Ayca A, Nihat S, Mehmet K. possible false positive heart fatty acid binding protein in patient who has a high level of creatinine kinase 2012; JAEM ;11:241-2.

Bawaskar HS, Bawaskar PH. Management of the cardiovascular manifestations of poisoning by the Indian red scorpion (Mesobuthus tamulus). Br Heart J 1992; 68:47880.

Bawaskar HS, Bawaskar PH. Management of scorpion sting. Heart 1999; 82: 253-4.

Ghani F, Wu AHB, Graff L, Petry C, Armstrong G, Prigent F, Brown M.(2000). Role of the heart type fatty acid binding protein in early detection of acute myocardial infarction. Clin Chem; 46:718-9.

Glatz JFC, van der Voort D, Hermens WT. Fatty acid binding protein as the earliest available plasma marker of acute myocardial injury. J Clin Ligand Assay 2002; 25(2): 167-77.

Hetland O, Dickstein K. Cardiac markers in the early hours of acute myocardial infarction: clinical performance of creatine kinase, 
creatine kinase MB isoenzyme (activity and mass concentration), creatine kinase $\mathrm{MM}$ and $\mathrm{MB}$ subform ratios, myoglobin and cardiac troponin T. Scand J Clin Lab Invest. 1996 Dec; 56(8):70113.

Himmatrao SB, Pramodini HB. Scorpion sting: update. JAPI 2012; 60:46-55.

Ho-Pang Yang, Fu-Chung Chen, Chien-Cheng Chen et al. Manifestations Mimicking Acute Myocardial Infarction after Honeybee Sting. Acta Cardiol Sin 2009; 25: 31-5.

Ishii J, Wang J, Naruse H, Taga S, Kinoshita M, Kurokawa $H$, Iwase M, Kondo T, Nomura M, Nagamura Y, Watanabe $Y$, Hishida H, Tanaka T, Kawamura K. Serum concentration of myoglobin vs human heart-type cytoplasmic fatty acid-binding protein in early detection of acute myocardial infarction. Clin Chem 1997; 43:1372-8.

Katrukha A, Bereznekiva A, Filatov V, Esakova T. Improved detection of minor ischemic cardiac injury in patients with unstable angina by measurement of TnI and FABP. Clin Chem 1999; 45:A139.

McCann CJ, Clover BM, Menowin IBA, et al. Novel biomarkers in early diagnosis of acute myocardial infarction compared with cardiac troponin T. Eur Heart J. 2008; 29:2843-2850.

O'Donoghue $M$, de Lemos JA, Morrow DA, Murphy SA, Buros JL, Cannon CP, et al. Prognostic utility of heart-type fatty acid binding protein in patients with acute coronary syndromes.

Circulation 2006; 114: 550-7.

Okamoto F, Sohmiya K, Ohkaru Y, Kawamura $K$, Asayama $K$, Kimura H, Nishimura S, Ishii H, Sunahara N, Tanaka T. Human heart-type cytoplasmic fatty acidbinding protein (H-FABP) for the diagnosis of acute myocardial infarction. Clinical evaluation of H-FABP in comparison with myoglobin and creatine kinase isoenzyme MB. Clin Chem Lab Med 2000; 38:231-8.

Rahav G, Weiss T. Scorpion sting induced pulmonary oedema. Scintigraphic evidence of cardiac dysfunction. Chest 1990; 97: 147880.

Ruzgar O, Bilge AK, Bugra Z, et al. The use of human heart-type fatty acid-binding protein as an early diagnostic biochemical marker of myocardial necrosis in patients with acute coronary syndrome, and its comparison with troponin- $\mathrm{T}$ and creatine kinase-myocardial band. Heart Vessels. 2006;21:309-314.

Sayre MR, Kaufmann KH, Chen I, et al. Measurement of cardiac troponin $\mathrm{T}$ is an effective method for predicting complications among emergency department patients with chest pain. Ann Emerg Med. 1998;31:539-549.

Seino Y, Ogata K, Takano T, et al. Use of a whole blood rapid panel test for heart-type fatty acidbinding protein in patients with acute chest pain: comparison with rapid troponin $\mathrm{T}$ and myoglobin tests. Am J Med. 2003;115:185190.

Tsuji R, TanakaT, SohmiyaK, HirotaY, Yoshimoto K, Kinoshita K, Kusaka Y, 
Kawamura K, Morita H, Abe S. Human heart type cytoplasmic fatty acid-binding protein in serum and urine during hyperacute myocardial infarction. Int J Cardiol 1993; 41:209-17.

Zarich SW, Qamar AU, Werdmann MJ, et al. Value of a single troponin $\mathrm{T}$ at the time of presentation as compared to serial CK-MB determinations in patients with suspected myocardial ischemia. Clin Chim Acta. 2001;326:185-192. 
الملخص العربى

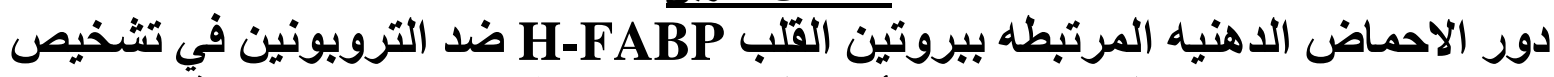

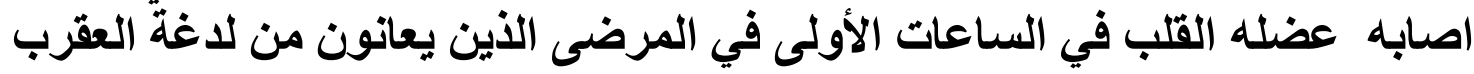

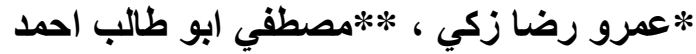

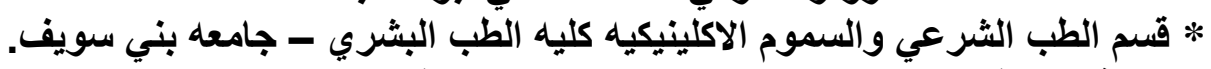

$$
\begin{aligned}
& \text { ** قسم الطب الثرعي والسموم الاكلينيكيه كليه الطب البشري - جامعل المنيا. }
\end{aligned}
$$

تمثل لاغه العقرب خطر ا على كبيرا علي الصحة العامة في المناطق الددارية الاسنو ائية وشبه الاستو ائيه. من الهن

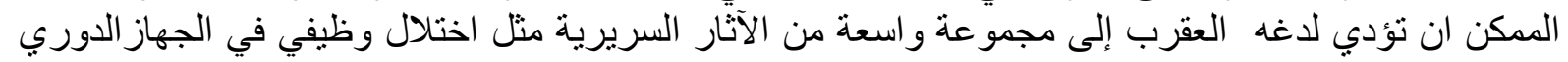

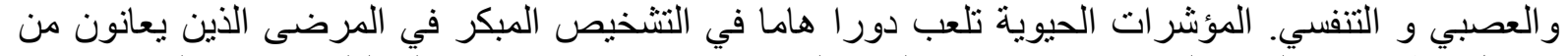

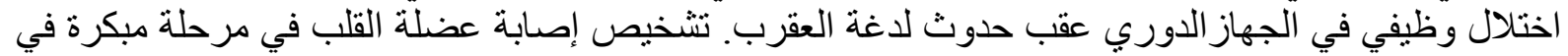

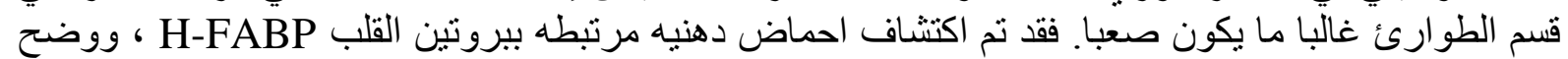

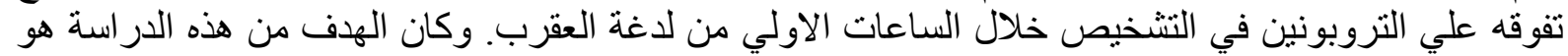

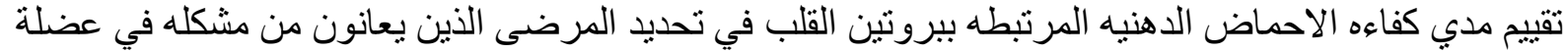

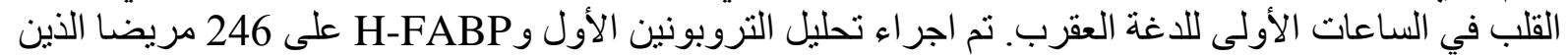

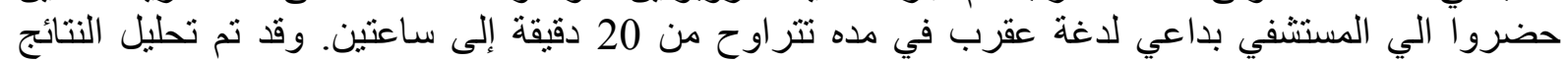

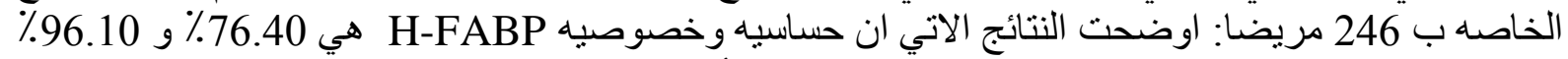

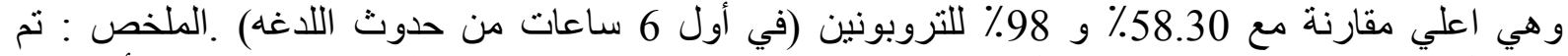

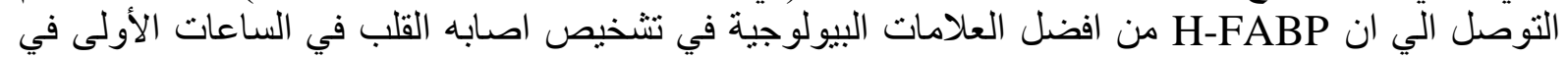
المرضى الذين بعانون من لدغة العقرب. 